\title{
Identificação do ruído ao longo dos turnos na terapia intensiva neonatal de hospital de ensino
}

\author{
Identification of noise throughout the shifts in neonatal intensive care of the teaching \\ hospital
}

\section{Identificación del ruido durante los turnos de cuidados intensivos neonatales en un hospital universitario}

Barsam, Fabiana Jorge Bueno Galdino ${ }^{\text {; }}$ Silva, Natália Yasmin Elisiário Barreto da²; Uramoto, Larissa Cristina Lima3; Teixeira, Cinthia Lorena Silva Barbosa4; Camargo, Fernanda Carolina5; Zullo, Sérgio Antônio ${ }^{6}$

Como citar este artigo: Barsam FJBG, Silva NYEB, Uramoto LCL, Teixeira CLSB, Camargo FC, Zullo AS. Identificação do ruído ao longo dos turnos na terapia intensiva neonatal de hospital de ensino. J. nurs. health. 2019;9(2):e199208

\section{RESUMO}

Objetivo: quantificar o ruído ao longo e após turnos de trabalho em uma unidade de terapia intensiva neonatal de um hospital público de ensino para controle ambiental. Métodos: estudo observacional, descritivo em unidade de terapia intensiva neonatal, referência para macrorregião de saúde em Minas Gerais. Foram realizadas quantificações do ruído em parceria com serviço de saúde ocupacional e segurança do trabalho a partir de quatro sessões de aferição entre agosto e setembro de 2017. Resultados: apesar da redução do ruído no período noturno, todos valores aferidos nos diferentes turnos encontravam-se acima dos valores confortáveis para o ambiente, medições entre 62 e $82 \mathrm{~dB}$. Maiores valores identificados devem-se pela: circulação de profissionais, docentes e discentes e necessidade de comunicação verbal. Conclusão: os valores das medições de ruído tem se apresentado acima de recomendações, similar às diferentes localidades mundiais, fato que exige medidas urgentes e sistemáticas para o seu controle.

Descritores: Monitoramento do ruído; Unidades de terapia intensiva neonatal; Conforto do paciente.

\section{ABSTRACT}

Objective: to quantify the noise along and after work shifts in a neonatal intensive care unit of a public teaching hospital for environmental control. Methods: observational, descriptive study in a

1 Médica. Doutora em Ciências da Saúde. Universidade Federal do Triângulo Mineiro (UFTM). E-mail: faturka2002@hotmail.com http://orcid.org/0000-0002-8143-4083

2 Discente do curso de Medicina. Universidade Federal do Triângulo Mineiro (UFTM). E-mail: natalia.elisiario@gmail.com http://orcid.org/0000-0003-0561-9796

3 Fonoaudióloga. Especialista em Voz. E-mail: larissalima_fonoaudiologia@hotmail.com http://0000-0003-14498789

4 Enfermeira. Especialista em Enfermagem em Unidade de Terapia Intensiva Neonatal. Hospital de Clínicas da Universidade Federal do Triângulo Mineiro (UFTM). E-mail: cinthiabarbosa.enf@gmail.com http: / /orcid.org/0000-0001-7864-5528

5 Enfermeira. Doutora em Atenção à Saúde. Empresa Brasileira de Serviços Hospitalares (EBSERH). E-mail: fernandaccamargo@yahoo.com.br http://orcid.org/0000-0002-1048-960X

6 Estatístico. Mestre em estatística. Empresa Brasileira de Serviços Hospitalares (EBSERH). E-mail: sergio.zullo@ebserh.gov.br http://orcid.org/0000-0002-7703-3172 


\section{JOURNALOF

neonatal intensive care unit, a reference for health macro - region in Minas Gerais. Noise quantification was carried out in partnership with occupational health and safety services from four calibration sessions between August and September 2017. Results: despite noise reduction in the night, all values measured in the different shifts were found above the comfortable values for the environment, measurements between 62 and $82 \mathrm{~dB}$. Higher values identified are: the circulation of professionals, teachers and students and the need for verbal communication. Conclusion: the values of noise measurements have been presented above recommendations, similar to different localities worldwide, a fact that requires urgent and systematic measures for their control.

Descriptors: Noise monitoring; Intensive care units, neonatal; Patient comfort.

\section{RESUMEN}

Objetivo: cuantificar ruido a largo y después de los turnos de trabajo en unidad de terapia intensiva neonatal de hospital público de enseñanza para control ambiental. Métodos: estudio observacional, descriptivo en unidad de terapia intensiva neonatal, referencia para macrorregión de salud en Minas Gerais. Se realizaron cuantificaciones del ruido em asociación con servicio de salud ocupacional y seguridad del trabajo a partir de cuatro sesiones de evaluación entre agosto y septiembre de 2017. Resultados: a pesar de reducción del ruido en período nocturno, todos valores aferrados en diferentes turnos se encontraban por encima de valores cómodos para el medio ambiente, mediciones entre 62 y $82 d B$. Mayores valores identificados se deben: circulación de profesionales, docentes y discentes y necesidad de comunicación verbal. Conclusión: valores de mediciones de ruido se han presentado por encima de recomendaciones, similar a diferentes localidades mundiales, hecho que exige medidas urgentes y sistemáticas para su control.

Descriptores: Monitoreo del ruido; Unidades de cuidado intensivo neonatal; Comodidad del paciente.

\section{INTRODUÇÃO}

0 ambiente da Unidade de Terapia Intensiva Neonatal e Pediátrica (UTINP), do qual a sobrevivência do Recém-Nascido (RN) de alto risco depende, é cercado de desafios à criança, aos seus pais e aos profissionais de saúde ali presentes, visto que o RN será exposto a um ambiente inóspito, com frequentes estímulos nocivos, como o estresse e a dor. ${ }^{1}$ Estudo demonstrou que cerca de 50\% dos pacientes de UTINP desenvolvem transtornos importantes do sono durante sua internação. 0 ruído diminui a duração e a quantidade dos períodos de sono Rapid Eye Moviment (REM), repercutindo no bem-estar psicológico e fisiológico dos pacientes críticos. Além disso, a exposição persistente de ruídos em excesso pode produzir perda auditiva, aumento da pressão intracraniana, estresse, hipertensão arterial, instabilidade metabólica, irritabilidade e perda do apetite, principalmente em prematuros. ${ }^{2}$

Desse modo, o local de terapia deve ser propício ao pleno restabelecimento desses $\mathrm{RN}$, uma vez que esta população possui maior risco de desenvolverem distúrbios cognitivos, motores e comportamentais em relação aos RNs a termo, sendo necessária a promoção do manuseio mínimo, assim como o controle dos fatores ambientais que provocam irritabilidade e ruídos excessivos. ${ }^{3-4}$

Historicamente, quanto ao controle dos ruídos no ambiente da UTINP, as primeiras pesquisas datam 
da década de 1970 no âmbito internacional, enquanto no Brasil, somente a partir da década de 1990 que se iniciaram as pesquisas sobre 0 tema. ${ }^{1}$

$\mathrm{Na}$ atualidade, $\mathrm{O}$ controle ambiental do ruído de UTINP continua a ser uma ação de interesse em hospitais de ensino em diferentes localidades mundiais. Estudos têm apontado valores aferidos acima dos limites de segurança recomendados, entre 35 e $45 \mathrm{~dB}$, como exemplificado por uma experiência norte-americana para controle do ruído em UTINP, cujas medições prévias à intervenção apresentaram valores médios de $\mathbf{5 7 . 0}$ $\mathrm{dB}$ (desvio padrão $\pm 0,84$ ). Em um hospital de ensino no México, as aferições revelaram uma média de 59.9 dB (desvio padrão $\pm 4,8$ ). Em um hospital canadense, um sistema de auditoria identificou limites entre 45 ou $55 \mathrm{~dB}$, no setor em questão do hospital. ${ }^{5-7}$

Os desafios para manutenção e o controle do ruído são ainda maiores em UTINP de hospitais públicos de ensino, por serem ambientes de elevada circulação de pessoas, onde convivem no mesmo espaço assistencial: estudantes, docentes, pesquisadores, familiares e trabalhadores de saúde. Desta maneira, a implementação de iniciativas que possibilitem o controle do ruído, a fim de se promover um ambiente de boas práticas assistenciais, de ensino e para a saúde do trabalhador tem se destacado como necessário. ${ }^{8-9}$

Mediante essa realidade, questiona-se sobre: qual a distribuição do ruído ao longo dos turnos em uma UTINP? Desta maneira, objetivou-se quantificar o ruído ao longo e após turnos de trabalho em uma unidade de terapia intensiva neonatal de um hospital público de ensino para controle ambiental.

\section{MATERIAIS E MÉTODOS}

Trata-se de um estudo observacional, descritivo sobre mensuração dos ruídos de uma UTINP composta por 20 leitos em hospital geral, público e de ensino, de grande porte (332 leitos) - referência macrorregional para a alta complexidade assistencial do polo Triângulo Sul de Minas Gerais, Brasil. Caracteriza-se por ser único hospital essencialmente público de alta complexidade na região e é referência em casos de obstetrícia de risco e no atendimento de RNs prematuros.

As medidas analisadas para a aferição do ruído no ambiente consideraram a estrutura física da UTINP, disposta em área assistencial, conforme salão único (complementar). A disposição da área e das demais estruturas físicas que compõem a unidade podem ser vistas na planta esquemática apresentada na Figura 1.

Registrou-se a ocorrência dos eventos sonoros emitidos pelas fontes de ruídos de alta e média frequência relatadas presentes na unidade. A quantificação consistiu em mensuração do ruído em diferentes espaços da UTINP por equipe especializada do Serviço de Saúde Ocupacional e Segurança do Trabalho (SOST) vinculado ao hospital de ensino.

0 ruído sonoro da unidade foi registrado e gravado, utilizando-se um dosímetro (Instrutherm ${ }^{\circledR}$ ), modelo 


\section{NURSING \\ AND HEALTH}

ISSN 2236 - 1987

DEC-46, devidamente calibrado. Foram avaliados os ruídos contínuos, em que os níveis de pressão sonora variam em mais ou menos $3 \mathrm{~dB}$, durante intervalos de tempo acima de 15 minutos, em sua maioria. Em cada um dos 42 pontos estabelecidos dentro da UTINP do hospital de ensino, efetuaram-se avaliações dos níveis de ruídos contínuo, medidos na escala de compensação "A" e velocidade de detecção Slow (devagar). Esse método de aferição do ruído apresenta-se conforme estabelecido por Associação Brasileira de Normas e Técnicas (ABNT). ${ }^{10}$ Foram realizadas quatro sessões de aferição no período de 18 de agosto de 2017 a 21 de setembro de 2017, distribuídas nos seguintes horários: $06 \mathrm{~h} 30 \mathrm{~min}$, 11h30min, $18 \mathrm{~h} 30 \mathrm{~min}$ e $21 \mathrm{~h} 00 \mathrm{~min}$ a fim de permitir a identificação das fontes geradoras de ruídos em diferentes momentos.

Figura 1: Planta esquemática da UTINP: 1, 3, 9, 10, 16, 18, 24, 25, 26, 27, 28, 33, 34, 35, 36, 37, 38, 39: incubadora; $2,7,17,22$ : cadeiras de reunião multidisciplinar; 4, 5, 6, 19, 20, 21, 40, 41, 42: mesa de reunião multiprofissional; 8, 31, 32: cadeira de prescrição; 11: posição esquerda do balcão de entrega de dieta e hemoderivados; 12: posição central do balcão de entrega de dieta e hemoderivados; 13: posição direita do balcão de entrega de dieta e hemoderivados; 14, 15, 23: pia de lavagem de mãos e/ou preparo de medicamentos; 29, 30: computadores.

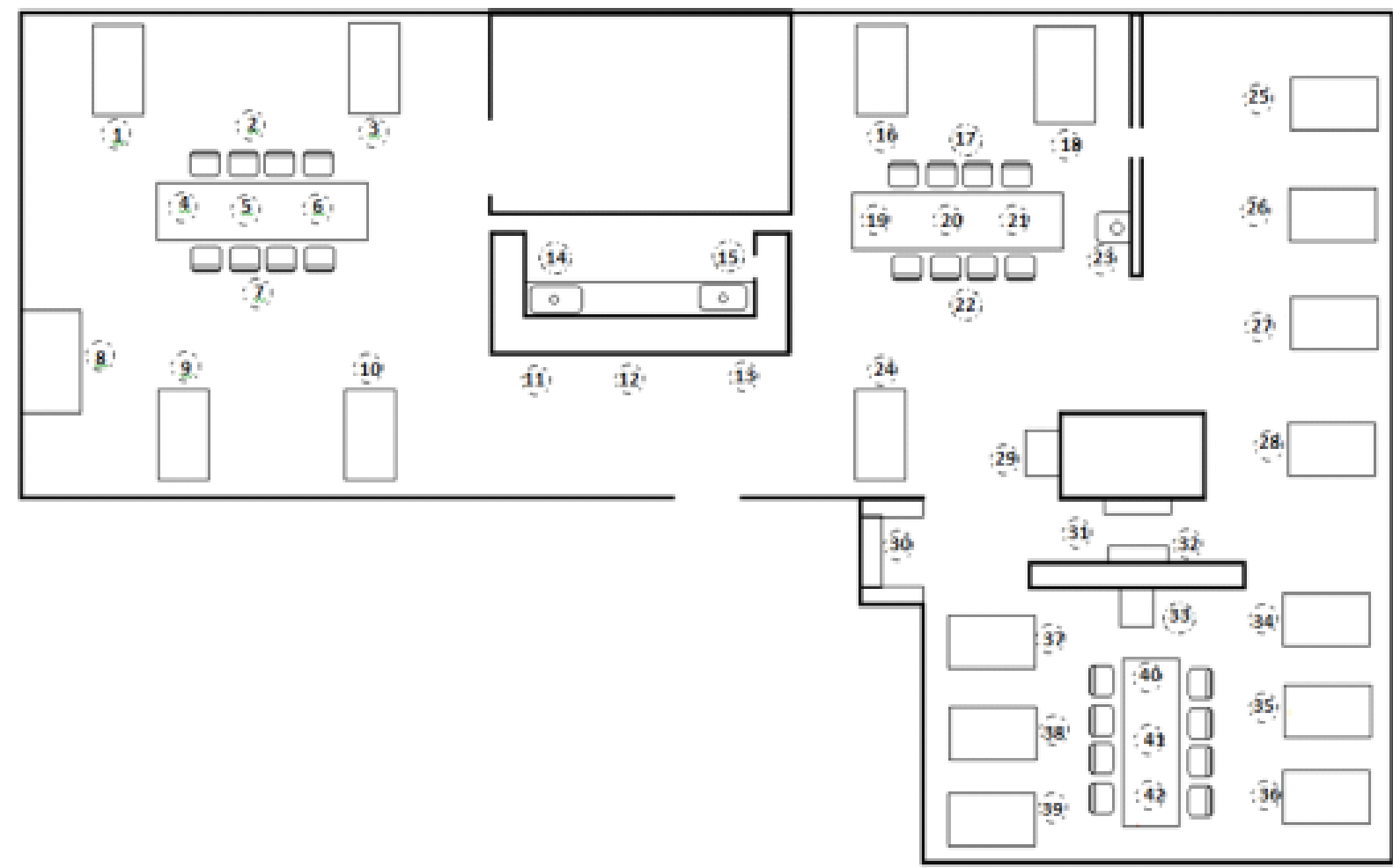

Fonte: Serviço de Saúde Ocupacional e Segurança do Trabalho do Hospital de Clínicas da Universidade Federal do Triângulo Mineiro, 2017

Para análise dos dados, organizou-se um banco de dados em Excel ${ }^{\circledR}$ por dupla digitação. $\quad \mathrm{Na}$ identificação de inconsistências foram acessados os roteiros para averiguação, a fim de assegurar o preenchimento adequado das 


\section{NURSING \\ AND}

ISSN $2236-1987$

informações. Os dados foram transportados para o programa estatístico Statiscal Package for Social Sciences (SPSS), versão 20.0 e Statistic versão 8.0. Realizada análise exploratória descritiva das mensurações por medidas de tendência central e dispersão. Ainda, calculada a média das diferenças entre os valores medidos e o valor máximo permitido para cada horário, denominado por diferença média. Sendo que para o horário das 06h30min, 11h30min, $18 \mathrm{~h} 30 \mathrm{~min}$ foi considerado como valor máximo permitido $45 \mathrm{~dB}$ e para o horário das $21 \mathrm{~h} 00 \mathrm{~min}$ o valor máximo foi de $35 \mathrm{~dB}$.

A coleta dos dados só foi iniciada após aprovação da presente proposta em Comitê de Ética em Pesquisa da Universidade Federal do Triangulo Mineiro sob número por extenso Certificado de Apresentação para Apreciação

Etica 58215416.7.0000.5154 e sob parecer $n$. 1.1618.872, ano 2017. Os membros da equipe e os familiares das crianças internadas foram informados quanto à natureza da pesquisa e assegurados de que suas vozes não seriam registradas, bem como de que não haveria qualquer tipo de registro que possibilitasse identificá-los.

\section{RESULTADOS}

Identificou-se que os valores aferidos nos diferentes turnos, de forma geral, encontravam-se acima dos valores confortáveis para o ruído em ambiente de UTINP. Há de se destacar que, apesar do horário das $21 \mathrm{~h}$ ter apresentado menor média aferida de ruído, a diferença-média é mais elevada, pois o valor de conforto esperado é de $35 \mathrm{db}$ (Tabela 1).

Quanto à distribuição dos valores de ruído aferido ao longo dos turnos, observa-se que há uma redução do ruído para o período noturno, entretanto, mediante aos valores sonoros confortáveis, preconizados todos os períodos, apresentam uma distribuição que excede esse valor (Figura 2).

Os grupos B e C agregam os maiores valores de aferição de ruído. Nestas áreas são dispostas duas mesas para reunião multidisciplinar $(19,20$, $21,40,41,42$ ), balcão de entrega de dieta e hemoderivados $(11,12,13)$ e incubadoras $(34,35,36,37,38,39)$. Os maiores valores identificados devemse a maior circulação de profissionais, docentes e discentes nos horários analisados, maior necessidade de comunicação verbal e uma predileção da equipe para habitar tais áreas.

Os locais com os menores valores de ruído estão dispostos em áreas mais isoladas, com presença de mesa única para reunião multiprofissional $(4,5,6)$ e incubadoras organizadas com maior distância entre si $(1,3,9,10)$ (Tabela 2).

Tabela 1:Valores aferidos conforme turnos e a diferença das médias identificadas. Brasil, 2017.

\begin{tabular}{cccc}
\hline Horário & Média & Desvio-padrão & Diferença-média \\
\hline $06 \mathrm{~h} 30$ & 64,83 & 4,87 & 19,83 \\
$11 \mathrm{~h} 30$ & 63,00 & 3,71 & 18,00 \\
$18 \mathrm{~h} 30$ & 63,79 & 1,99 & 18,79 \\
$21 \mathrm{~h} 00$ & 56,40 & 2,93 & 21,40 \\
\hline
\end{tabular}

Fonte: dados da pesquisa, 2017. 
Figura 2: Distribuição dos valores de ruídos conforme quantificação geral por turnos por média, valor mínimo e máximo das aferições por área e demais estruturas físicas. Brasil, 2017.

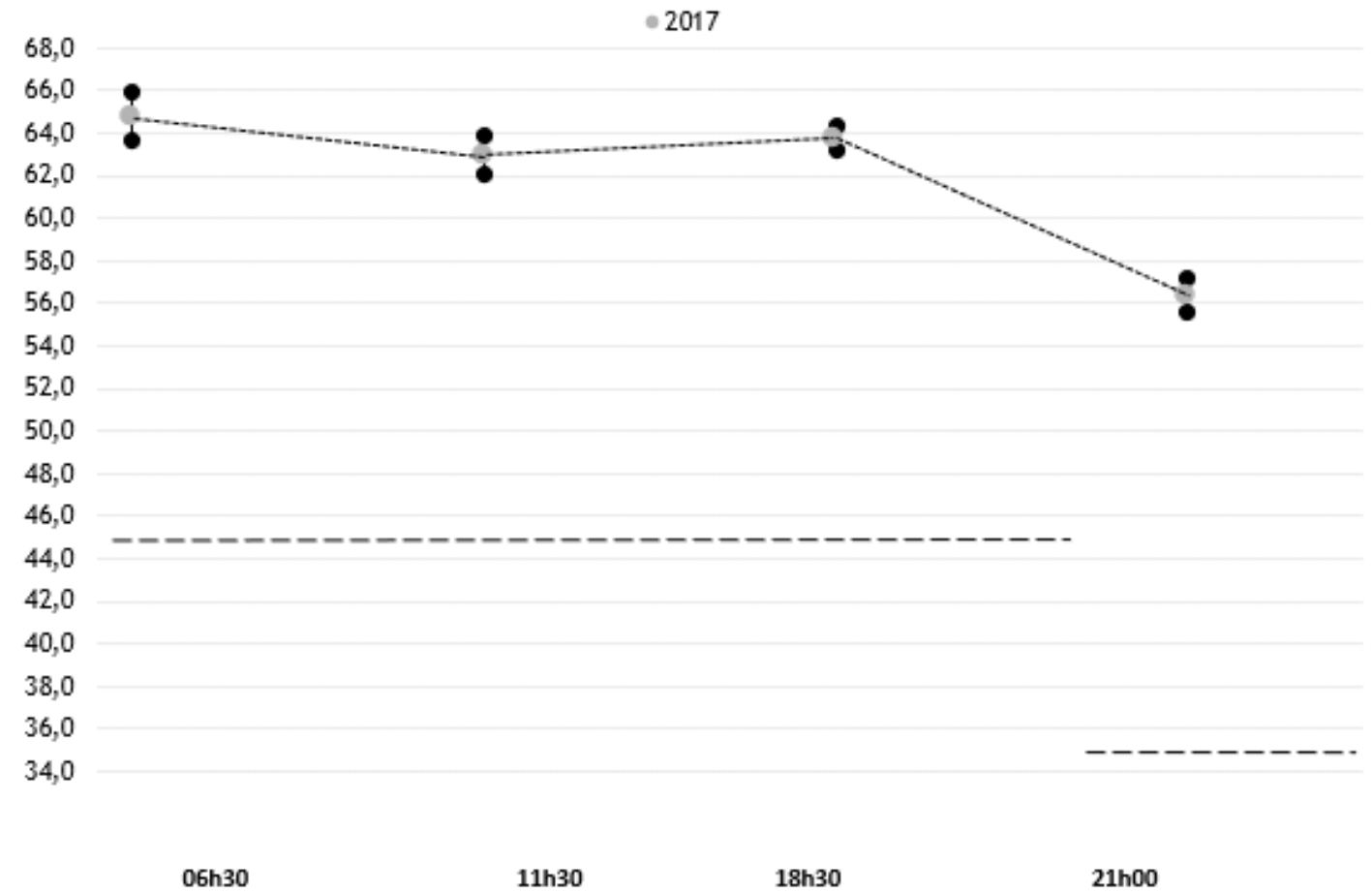

HORÁRIOS

Obs: as linhas tracejadas referem aos valores seguros para controle do ruído nos períodos analisados. Fonte: dados da pesquisa, 2017.

Tabela 2: Descrição da quantificação de ruídos por grupos conforme turnos e aferições por área e demais estruturas físicas. Brasil, 2017.

\begin{tabular}{|c|c|c|c|c|c|c|c|c|c|}
\hline \multirow{3}{*}{ Grupos } & \multirow{3}{*}{ Áreas } & \multicolumn{8}{|c|}{ Horários de Aferição } \\
\hline & & \multicolumn{2}{|c|}{$06 \mathrm{~h} 30$} & \multicolumn{2}{|c|}{$11 \mathrm{~h} 30$} & \multicolumn{2}{|c|}{$18 \mathrm{~h} 30$} & \multicolumn{2}{|c|}{$21 \mathrm{~h} 00$} \\
\hline & & Média & IC95\% & Média & IC95\% & Média & IC $95 \%$ & Média & IC95\% \\
\hline A & $\begin{array}{c}4 ; 5 ; 6 ; 7 ; \\
10 ; 14 ; 15 ; \\
16 ; 17 ; 18 ; \\
22 ; 25 ; 26 ; \\
27 ; 29 ; 31 ; \\
32 ; 33\end{array}$ & 64,9 & $\begin{array}{c}64,2: \\
65,7\end{array}$ & 61,3 & $\begin{array}{c}60,4: \\
62,3\end{array}$ & 64,3 & $\begin{array}{c}63,3: \\
65,4\end{array}$ & 55,2 & $\begin{array}{c}54,7: \\
55,8\end{array}$ \\
\hline B & $\begin{array}{c}1 ; 2 ; 3 ; 9 ; \\
19 ; 21 ; 23 \\
24 ; 28 ; 30 ; \\
34 ; 35 ; 37 \\
39\end{array}$ & 60,0 & $\begin{array}{c}59,0: \\
61,0\end{array}$ & 62,5 & $\begin{array}{c}60,8: \\
64,2\end{array}$ & 63,1 & $\begin{array}{c}62,0: \\
64,1\end{array}$ & 57,0 & $\begin{array}{c}55,3: \\
58,7\end{array}$ \\
\hline C & $\begin{array}{c}8 ; 11 ; \\
12 ; 13 ; 20 ; \\
36 ; 38 ; 40 ; \\
41 ; 42\end{array}$ & 71,4 & $69,2: 73,6$ & 66,7 & $64,1: 69,3$ & 63,8 & $63,2: 64,4$ & 57,7 & $55,2: 60,2$ \\
\hline
\end{tabular}

Fonte: dados da pesquisa, 2017. 


\section{DISCUSSÃO}

0 presente estudo obteve em todas as aferições níveis de ruído acima do preconizado ao ambiente UTINP nos diferentes períodos de aferição. Em muito, o controle do ruído em UTINP é ação de interesse em diversos hospitais de ensino do mundo, uma vez que diferentes estudos demonstraram valores sonoros acima dos recomendados para cenários hospitalares onde há integração ensino-serviço. ${ }^{11-12}$

Os desafios impostos ao cenário nacional para o controle adequado do ruído em UTINP são complexos, pois permeiam desde a necessidade em ser superar práticas organizacionais tradicionais - como a hierarquização rígida para a tomada de decisões - até a necessidade de promover 0 engajamento das equipes na adoção de medidas para o controle deste. Essas seriam iniciativas cruciais para que alcance um ambiente favorável a uma cultura de segurança do paciente quanto ao manejo adequado do ruído. ${ }^{11-13}$

Adicionalmente, outro desafio apresenta-se pelo avanço e pela sofisticação tecnológica, necessários para uma assistência de qualidade em ambiente hospitalar, que geram altos níveis de poluição sonora, tornando o ambiente perturbador e contribuindo para o desenvolvimento de alterações fisiopatológicas tanto nos pacientes quanto nos profissionais presentes. ${ }^{11-13}$

Haja vista que em decorrência dessa realidade, essa tendência associada principalmente ao surgimento de dispositivos médicos
ISSN 2236 - 1987

com alarmes sonoros, provocou nas últimas décadas um incremento nos níveis de ruídos das UTINP. Exemplifica-se 0 fato, conforme revisão da literatura quando em 1960 ruídos aferidos eram de $57 \mathrm{~dB}$ durante o dia e $42 \mathrm{~dB}$ durante 0 período noturno, para UTINP e em 2005 essas mediações variam de $72 \mathrm{~dB}$ no dia e 60 $\mathrm{dB}$ a noite. ${ }^{13}$

Entretanto, Organização Mundial da Saúde recomenda, para a UTINP, níveis de pressão sonora de até $40 \mathrm{~dB}$ durante o dia, com redução de 5 a 10 $\mathrm{dB}$ à noite. ${ }^{12-13}$ Corroborando com os achados do presente estudo, pesquisa desenvolvida em hospital público do município do Rio de Janeiro aferiu o nível de pressão sonora ambiente em uma UTINP e evidenciou valores além do ideal e tolerável em todas as aferições, ultrapassando em 50\% o valor indicado como conforto (35dB). Os equipamentos com alarmes acústicos, a conversação de profissionais e familiares e o choro do $\mathrm{RN}$ foram os maiores responsáveis pelo ruído excessivo. ${ }^{14}$

Revisão sistemática da literatura evidenciou que a exposição contínua a ruídos acima dos valores de conforto prejudica 0 desenvolvimento do neonato. Níveis de ruído entre 55 e 65 $\mathrm{dB}$ podem produzir excitações nervosas e estresse, tornando o RN mais suscetível a dor e outros desconfortos. Os maiores níveis sonoros registrados no ambiente da UTINP advém do período diurno, quando são realizados controles médicos, procedimentos eletivos, visitas de equipes multiprofissionais e familiares, atividades didáticas características de um hospital de ensino, alimentação, 


\section{JOURNALOF

entre outras práticas. Além disso, níveis excessivos de ruídos provocam alterações na equipe, potencializando a ocorrência de síndrome de Burnout, por exemplo. ${ }^{15}$

Em unidades de terapia intensiva chinesas os alarmes de monitores $(98,3$ $\mathrm{dB})$, a troca de plantão $(91 \mathrm{~dB})$ e a conversa da equipe $(88,5 \mathrm{~dB})$ foram as fontes de mais elevado ruído provocado no ambiente. ${ }^{16}$

Para a superação dessa realidade, torna-se eminente induzir mudanças de comportamento. Há uma necessidade contínua de reforçar as práticas de cuidado e ambientes assistenciais seguros aos pacientes e equipe de saúde. A educação continuada sobre os efeitos do som, mudanças nas práticas de cuidados operacionais para reduzir a exposição a níveis sonoros excessivos, juntamente com lembretes que garantam a manutenção do silêncio e monitoramento dos níveis sonoros, de forma contínua ou intermitente, apresentam potencial para melhorar 0 controle do ruído em na UTINP. ${ }^{15-17}$

A retirada de equipamentos como latas de lixo metálicas, cestos e telefones com toques são outras formas econômicas para reduzir os níveis sonoros, caso uma renovação completa não seja viável. Pais engajados como membros da equipe continuam sendo a chave para a sustentabilidade. 0 fornecimento de educação sobre redução sonora, encorajando vozes sussurrantes, assegurando tempo de silêncio com o $\mathrm{RN}$, alertando a equipe quanto ao monitoramento de som, e silenciando telefones celulares são outras práticas para reduzir o ruído prejudicial na UTINP. ${ }^{15-17}$

Quantos as limitações do presente estudo, trata-se de uma análise de aferição única o que impede a observação de variações e a identificação de um valor constante quanto à quantificação do ruído no ambiente. Entretanto, o método de aferição empreendido considera a rotina do SOST local, o que possibilitou uma aproximação e um diagnóstico inicial do cenário. Os resultados apresentam-se como dados preliminares, sendo eles de suma importância para a realidade local e o seu enfrentamento pelo desenvolvimento de programas.

\section{CONCLUSÃO}

De acordo com os resultados do presente estudo com medição do ruído entre 62 e $82 \mathrm{~dB}$, o controle do ruído tem se apresentado como um desafio para a UTINP de um hospital de ensino, similar a diferentes localidades mundiais. Os valores das medições de ruído têm se apresentado acima de recomendações para um cenário seguro aos neonatos e à saúde dos trabalhadores. Fato que exige medidas urgentes e sistemáticas para o seu controle.

Novas pesquisas devem ser empreendidas para identificar demais fatores que contribuíram o adequado gerenciamento do ruído, como também para a motivação e satisfação dos trabalhadores, estudantes e docentes em integrarem o cenário do hospital de ensino, visando o melhor controle desse aspecto. Também seriam importantes pesquisas longitudinais sobre como as iniciativas 
contribuíram para a redução do ruído e o bem-estar dos trabalhadores.

\section{REFERÊNCIAS}

1 Santana LSR, Silva LS, Silva RR, Carvalho JE, Santana WS, RossiBarbosa LAR, et al. Quantificação dos ruídos sonoros em uma unidade de terapia intensiva neonatal. REME rev. min. enferm. [Internet]. 2015 abr/jun[acesso em 2019 jun 28];19(2):27-31. Disponível em: https://doi.org/10.5935/14152762.20150023

2 Galindo APG, Caicedo YC, VélezPereira AM. Nivel de ruído en unidades de cuidado intensivo de un hospital público universitário en Santa Marta (Colombia). Med. intensiva. (Madr., Ed. Impr.) [Internet]. 2016[acesso em 2019 set 18];40(7):403-10. Disponível em:

https://www.sciencedirect.com/scien ce/article/abs/pii/S021056911500272 7

3 Chawla S, Barach P, Dwaihy M, Kamat D, Shankaran S, Panaitescu B, et al. A targeted noise reduction observational study for reducing noise in a neonatal intensive unit. J. Perinatol. [Internet]. 2017 Sept[cited 2019 Sept 18];37(9):1060-4. Available from: https://www.nature.com/articles/jp2 01793

4 Coelho AS, Custódio DCGG, Rosso G, Silva R, Silva JSC, Carniel F. Equipe de enfermagem e a assistência humanizada na UTI neonatal. Revista Ciência \& Saberes [Internet]. 2018[acesso em 2019 jun 28];4(1):873$7 . \quad$ Disponível em: http://www.facema.edu.br/ojs/index
.php/ReOnFacema/article/view/381/ 176

5 Silva EMB, Ramos ACFS, Duarte JC, Silva DM. Noise in neonatology: perception of health professionals. Referência. [Internet]. 2019[cited 2019 Jun 28];4(20):67-76. Available from:

https://doi.org/10.12707/RIV18078

6 Barsam FJBG, Teixeira CLSB, Oliveira CR, Lima LCS, Ferreira DO, Silva MSS, et al. Gerenciamento de mudanças para controle do ruído na terapia intensiva neonatal: relato de experiência. REME rev. min. enferm. [Internet]. 2019[acesso em 2019 jun 28];23:e-1154. Disponível em: http://www.reme.org.br/artigo/detal hes $/ 1295$

7 Nieto-Sanjuanero A, Quero-Jiménez J, Cantú-Moreno D, RodríguezBalderrama I, Montes-Tapia F, RubioPérez N, et al. Evaluation of strategies aimed at reducing the level of noise in different areas of neonatal care in a tertiary hospital. Gac. méd. Mex. [Internet]. 2015 Nov-Dec[cited 2019 Jun 28];151(6):741-8. Available from: http://www.anmm.org.mx/GMM/2015 /n6_english/2331AX156_151_2015_UK 6_687-694.pdf

8 Abreu RMD, Stacciarini TGS, Paula GF, Camargo FC, Garcia LAA, Pereira GA. Produção científica da equipe de enfermagem de um hospital público de ensino. Revista Família, Ciclos de Vida e Saúde no Contexto Social [Internet]. 2017[acesso em 2019 jun 28];5(3):390$7 . \quad$ Disponível em: http://seer.uftm.edu.br/revistaeletro nica/index.php/refacs/article/view/2 $136 /$ pdf 


\section{ISSN 2236 - 1987}

9 Lorenzetti J, Gelbcke FL, Vandresen L. Management technology for hospital inpatient care units. Texto \& contexto enferm. [Internet]. 2016[cited 2019 Jun 28]; 25(2):e1770015. Available from:

http://www.scielo.br/pdf/tce/v25n2/ 0104-0707-tce-25-02-1770015.pdf

10 Associação Brasileira de Normas Técnicas (ABNT). NRB 10151: acústica avaliação de ruído em áreas habitadas, visando o conforto da comunidade procedimento [Internet]. 2000[acesso em 2019 set 18]. Disponível em: http://www.sema.df.gov.br/wpconteudo/uploads/2017/09/NBR10151-de-2000.pdf

11 Camargo FC, Iwamoto HH, Galvão CM, Monteiro DAT, Goulart MB, Garcia LAA. Models for the implementation of evidence-based practice in hospital based nursing: a narrative review. Texto \& contexto enferm. [Internet]. 2017[cited 2019 Jun 28];26(4):e2070017. Available from: http://www.scielo.br/pdf/tce/v26n4/ en_0104-0707-tce-26-04-e2070017.pdf

12 Barbosa RT, Cruz ICF. Prática interprofissional de enfermagem baseada em evidência sobre déficit no autocuidado para vestir-se em UTI: revisão sistematizada da literatura. Journal of Specialized Nursing Care [Internet]. 2019[acesso em 2019 jun 28];11(19). Disponível em: http://www.jsncare.uff.br/index.php /jsncare/article/view/3130/790

13 Konkani A, Oakley B. Noise in hospital intensive care units - a critical review of a critical topic. J. crit. care. [Internet]. 2012 Oct[cited 2019 Sept 18];27(5): 522.e1-522.e9. Available from: https://doi.org/10.1016/j.jcrc.2011.0 9.003

14 Nogueira MFH, Ramos EG, Peixoto $M V M$. Identificação de fontes de ruído e de pressão sonora em unidade neonatal. Rev. enferm. UERJ. [Internet]. 2011[acesso em 2019 jun 28];19(4):517-23. Disponível em: http://www.facenf.uerj.br/v19n4/v1 9n4a02.pdf

15 Almadhoob A, Ohlsson A. Sound reduction management in the neonatal intensive care unit for preterm or very low birth weight infants. Cochrane database syst. rev. (online). [Internet]. 2015Jan[cited 2019 Jun 28];1:CD010333. Available from: https: / /www.cochranelibrary.com/cd sr/doi/10.1002/14651858.CD010333.p ub2/full

$16 \mathrm{Hu} \mathrm{RF}$, Hegadoren $\mathrm{KM}$, Wang $\mathrm{XY}$, Jiang $X Y$. An investigation of light and sound levels on intensive care units in China. Aust. crit. Care. [Internet]. 2016 May[cited 2019 Sept 18];29(2):627. https://doi.org/10.1016/j.aucc. 2015 . 08.001

17 Santos BR, Orsi KCSC, Balieiro MMFG, Sato MH, Kakehashi TY, Pinheiro EM. Effect of quiet time to reduce noise at the neonatal intensive care unit. Esc. Anna Nery Rev. Enferm. [Internet]. 2015[cited 2019 Jun 28];19(1):102-6. Available from: http://www.scielo.br/pdf/ean/v19n1 /en_1414-8145-ean-19-01-0102.pdf

Data de submissão: 28/06/2018

Data de aceite: 03/09/2019

Data de publicação: 08/10/2019 